\title{
Arquitectura y tecnología constructiva en los sitios arqueológicos El Obraje 01 y El Obraje 02, San Agustín de Cajas, Huancayo
}

\author{
Architecture and constructive technology in archaeological sites El Obraje 01 and \\ El Obraje 02, San Agustín de Cajas, Huancayo
}

Manuel F. Perales Munguía ', Hugo Marroquín Quijandría ${ }^{2}$ Universidad Continental

\section{RESUMEN}

Objetivos: Describir las características de la arquitectura y tecnología constructiva presente en los sitios arqueológicos El Obraje 01 y El Obraje 02 (distrito de San Agustín de Cajas, Huancayo), así como su estado de deterioro y sus tipos de lesiones. Métodos: Investigación básica, de alcance exploratorio-descriptivo y diseño transversal; efectuada mediante reconocimientos de campo, relevamientos topográficos y levantamientos arquitectónicos, así como registros fotográficos, identificación petrográfica y ensayos químicos de laboratorio sobre muestras de argamasa arqueológica. En gabinete se procesó la información en computadoras, con el uso del software AutoCad, Microsoft Excel, entre otros. Resultados: La arquitectura estudiada se caracteriza por un sistema espacial basado en la planta circular, con edificios asociados a espacios abiertos, sin patrón claro de organización. El sistema de circulación se compone de un recorrido principal continuo y flexible, carente de patrón alguno, mientras que el sistema estructural es portante. El estado de deterioro es moderado en El Obraje 02 y malo en El Obraje 01. Se identificaron lesiones mecánicas y químicas, mas no de naturaleza física. Conclusiones: La arquitectura estudiada muestra conceptos y principios relacionados a una cosmovisión no occidental. La tecnología constructiva revela una organización laboral sencilla y conocimientos notables de materiales y técnicas. La ausencia de lesiones físicas sugiere la eficacia de la tecnología constructiva y el sistema estructural, mientras que la existencia de lesiones mecánicas se

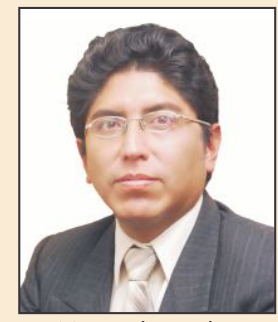

Manuel Perales

debe a recientes intervenciones de reconstrucción. Las lesiones químicas pueden ser consecuencia del paso del tiempo y la acción de la intemperie.

Palabras clave: Arquitectura, tecnología constructiva, monumentos arqueológicos, El Obraje, San Agustín de Cajas.

1 Arqueologo con estudios de Maestria en Antropología Jurídica, Docente de la Universidad Continental.

2 Arquitecto, Docente de la Universidad Continental. 


\section{ABSTRACT}

Objectives: To describe the characteristics of the architecture and the current constructive technology in the archaeological sites the Obraje 01 and the Obraje 02 (San Agustin de Cajas district, Huancayo) and its deterioration state and damage types. Methods: Basic research, with exploratorydescriptive scope and cross-sectional design, made by ground recognition, topographic surveys, architectural lifting and photographic records, petrographic identification and laboratory chemical trials on archaeological mortar samples. Enclosed was processed the information on computers, using AutoCad, Microsoft Excel, and other software. Results: The studied architecture is characterized by a spatial system based on the circular plant, with buildings associated to open spaces, without clear organization pattern. The circulation system is comprised of a flexible and continuous main path, lacking of any pattern, while the structural system is load bearing. The deterioration state is moderate in the Obraje 02 and bad in the Obraje 01. Mechanical and chemical damages were identified, but not of physical nature. Conclusions: The studied architecture shows concepts and principles related to a nonwestern worldview. The constructive technology reveals a simple labor organization and remarkable knowledge about materials and techniques. The absence of physical damage suggests the constructive technology effectiveness and the structural system, while the existence of mechanical damage due to recent reconstruction interventions. The chemical damages may be due to the passage of time and the effects of the weather.

Keywords: Constructive technology architecture, archaeological monuments, the Obraje, San Agustín de Cajas.

\section{INTRODUCCIÓN}

Durante los últimos años se ha generado en el Perú una expectativa importante acerca de los beneficios que el patrimonio arqueológico puede ofrecer al desarrollo del turismo. Precisamente, la asignación de partidas económicas procedentes del presupuesto público para proyectos de conservación y restauración de monumentos arqueológicos emblemáticos del país como Caral o Kuélap, a través de las denominadas unidades ejecutoras del Ministerio de Cultura, expresan esta tendencia.

En el Valle del Mantaro todavía no se han desarrollado experiencias de gran envergadura en relación con el aprovechamiento responsable del patrimonio arqueológico con fines turísticos, aunque actualmente ya se cuenta con perfiles y expedientes técnicos de proyectos de ese tipo, declarados viables en el Sistema Nacional de Inversión Pública (SNIP). Sin embargo, el desconocimiento sobre el tema, de parte de autoridades, funcionarios y consultores, ha llevado a que tales estudios no consideren, en varios casos, las pautas y recomendaciones (1) de instituciones y organismos nacionales e internacionales, como el Ministerio de Cultura del Perú, ICOMOS y la UNESCO, entre otros.

En este contexto, los discursos que califican a la actividad turística como panacea para salir de la pobreza, han nutrido una expectativa sobredimensionada en algunos sectores de nuestra población local en relación a este tema. Así, una de las consecuencias que este fenómeno ha traído es la destrucción de numerosos sitios arqueológicos del valle del Mantaro, en el afán de acondicionarlos para el turismo mediante reconstrucciones que carecieron totalmente de criterios especializados. Éste es el caso, por ejemplo, de los yacimientos arqueológicos de Chuctoloma (Quilcas, Huancayo), Ocollacta (Santa Rosa de Ocopa, Concepción), Chuctunmarca (San Juan de Jarpa, Chupaca) y El Obraje (San Agustín de Cajas, Huancayo). 
En el caso del último sitio mencionado, el año 2008 éste fue objeto de una reconstrucción no autorizada, a cargo del llamado Comité de Gestión del Proyecto Construcción del Vivero Forestal San Agustín, que contó con el financiamiento del Programa Construyendo Perú. Como era de esperarse, esta intervención alteró las características originales de las edificaciones prehispánicas del lugar y se disturbaron los depósitos culturales allí existentes con la remoción masiva de tierra destinada para los nuevos muros.

Frente a esta situación, consideramos pertinente iniciar estudios que permitan identificar las características de la arquitectura y la tecnología constructiva existentes en los sitios arqueológicos del valle del Mantaro, con la intención de ofrecer datos imprescindibles para la elaboración y ejecución de proyectos de conservación y restauración de nuestro patrimonio prehispánico, tengan éstos fines turísticos o no. Al mismo tiempo, creímos necesaria una evaluación de los daños ocasionados por aquellas reconstrucciones inconsultas, con la intención de recuperar información que facilite futuras acciones orientadas a mitigar los efectos negativos de tales trabajos. Para todo esto se eligió al sitio arqueológico El Obraje 02 y también, por su cercanía con respecto a éste, a El Obraje 01, yacimientos ambos que fueron declarados como Patrimonio Cultural de la Nación mediante Resolución Directoral Nacional Nro. 925/INC del ex - Instituto Nacional de Cultura (hoy Ministerio de Cultura), el año 2001.

Las investigaciones previas efectuadas en $\mathrm{El}$ Obraje 01 y El Obraje 02 son bastante escasas. Entre éstas, la contribución más importante fue desarrollada por David Browman (2), quien registró ambos sitios hacia fines de la década de 1 960, como parte de un estudio sobre la historia cultural del valle del Mantaro. En el sistema de nomenclatura empleado por Browman, El Obraje 01 fue catalogado con el código PJuM 747, mientras que El Obraje 02 se identificó como PJuM 662. Ambos yacimientos fueron ubicados cronológicamente por este autor dentro de la época que él denomina Arhuaturo-Inca, que corresponde al periodo Horizonte Tardío (ca. 1450 - 1533 d.C.) de la secuencia cultural prehispánica formulada para los Andes Centrales (3).

Los estudios sobre la arquitectura prehispánica en el valle del Mantaro son igualmente pocos, entre los cuales destaca el trabajo de Elizabeth DeMarrais (4) efectuado en los asentamientos prehispánicos tardíos del valle de Yanamarca, Jauja. En ellos, según muestra dicha autora, la unidad arquitectónica básica es el edificio de planta circular, que junto a otros similares suele aparecer asociado a espacios irregulares sin techar, a los cuales se accede a través de especies de corredores sinuosos, sin patrón claro de organización. De acuerdo a sus datos, se aprecian espacios públicos como plazas únicamente en los sitios de mayor extensión como Tunanmarca.

En cuanto a la tecnología constructiva, DeMarrais (4) reporta que las estructuras fueron levantadas con muros de doble hilera de piedras procedentes de canteras locales. Tales piedras exhiben poca modificación y fueron asentadas con mortero de barro, aunque no se describe su composición. Reportes de evidencias arquitectónicas similares se encuentran en las publicaciones de varios autores $(5,6,7,8,9)$ sobre otros sitios prehispánicos tardíos en la sierra central peruana.

Según la información que manejamos, los trabajos de Browman (2) en El Obraje 01 y El Obraje 02 incluyeron únicamente observaciones a nivel de superficie y recolecciones de material cultural visible sobre el terreno. Este autor no llevó a cabo excavaciones y otras labores, razón por la cual nuestra investigación tiene el mérito de haber efectuado, por primera vez, un estudio en detalle de las edificaciones que se hallan en pie en dichos lugares. Del mismo modo, los análisis de argamasa arqueológica que se han llevado a cabo son novedosos para la arqueología de esta parte del país, así como la metodología empleada 
para determinar el estado de deterioro de los sitios. Sin embargo, también debemos señalar que la falta de laboratorios especializados en procedimientos arqueométricos en la región limitó drásticamente la realización de análisis más profundos de las muestras de argamasa arqueológica. Además, el estado de deterioro más avanzado de las edificaciones en El Obraje 01 hizo que, finalmente, optásemos por focalizar nuestra atención en la arquitectura de El Obraje 02.

\section{MATERIAL Y MÉTODOS}

Como ya se indicó, los sitios arqueológicos conocidos con los nombres de El Obraje 01 y El Obraje 02 se encuentran en el distrito de San Agustín de Cajas, provincia de Huancayo, a 200 metros al este del barrio de Hatun Cajas (Figura 01). Ambos yacimientos ocupan la sección inferior de laderas de cerro que caen sobre el fondo del valle y que presentan suelos poco profundos

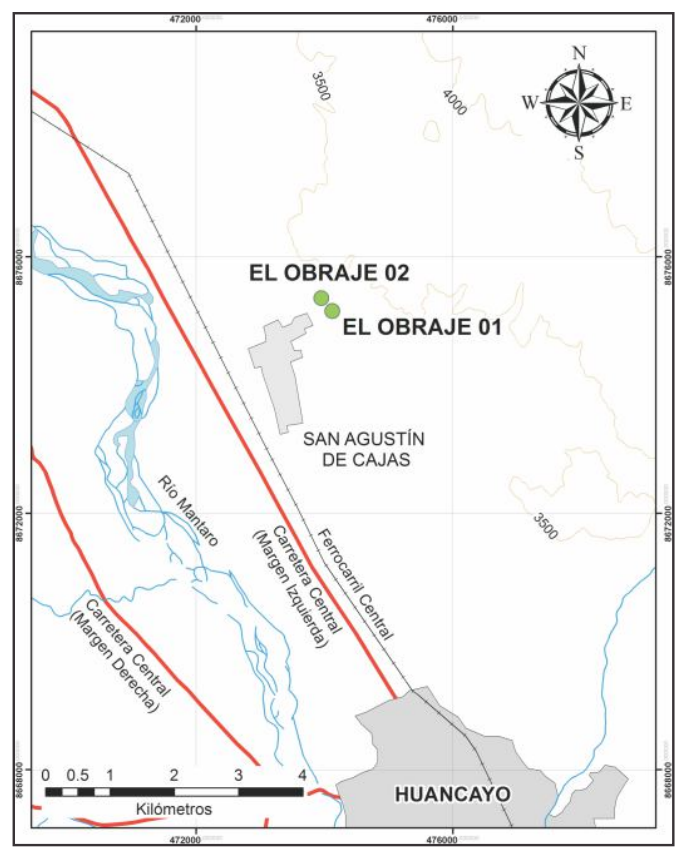

Figura 01. Mapa de ubicación de los sitios arqueológicos El Obraje 01 y El Obraje 02 (basado en la Carta Nacional IGN, hojas 24-m, 25-m; esc. 1:100 000. Intervalo de grilla: $4000 \mathrm{~m}$; esc. de publicación: 1: $50000)$. sobre la roca madre. Su extensión oscila entre 0,92 y 1,25 hectáreas y están conformados por aglomeraciones pequeñas de estructuras de piedra de planta circular. En El Obraje 01 resulta difícil determinar la cantidad de dichas edificaciones, en tanto que en El Obraje 02 se aprecian en forma clara veinte, la mayoría de las cuales fueron reconstruidas en el año 2008 (Figura 02, 03).

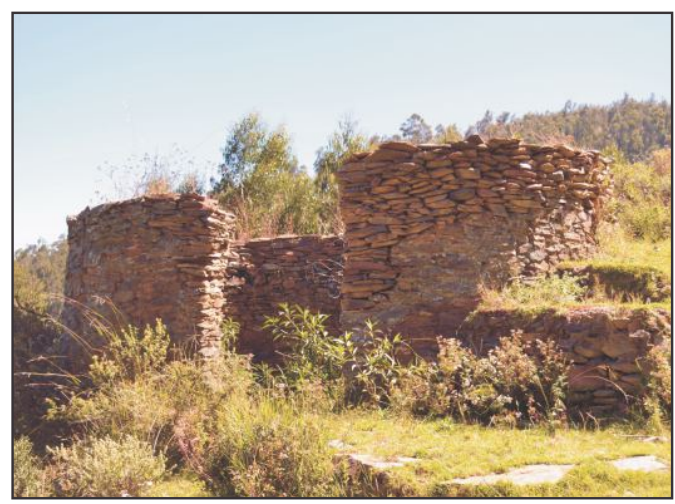

Figura 02: Vista frontal del edificio 02 en El Obraje 02, desde el este. Nótese la reconstrucción de los muros hacia su parte superior.

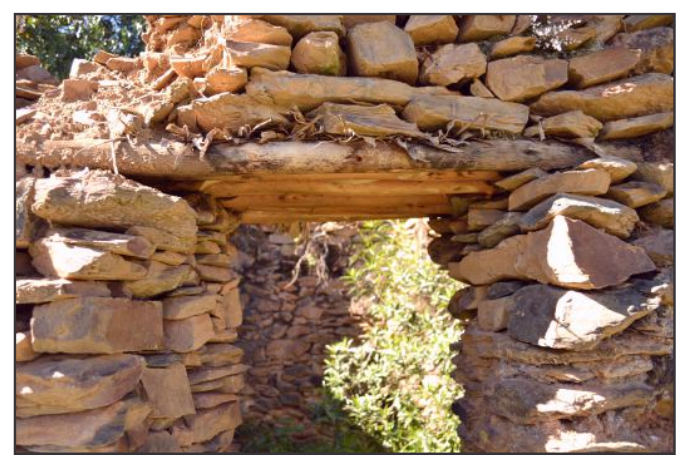

Figura 03: Detalle de la reconstrucción del vano de ingreso al edificio 09 en El Obraje 02. Nótese en el dintel el uso de ramas de eucalipto, árbol inexistente en tiempos prehispánicos en la región.

El carácter elusivo de la arquitectura prehispánica andina nos llevó a elegir, para nuestros estudios, un sitio de poca extensión, con la idea de identificar sus principios y bases conceptuales en un contexto de menor complejidad, según la pauta metodológica del "sitio pequeño" propuesta por Moseley y Mackey (10). Asimismo, en base a los criterios planteados por otros autores (11, $12,13,14)$, se optó por estudiar la 
arquitectura y tecnología constructiva en función de tres variables (sistema espacial, sistema de circulación, sistema estructural), mientras que para la descripción del estado de deterioro de los edificios se consideraron tres tipos de lesiones (físicas, mecánicas y químicas).

Los primeros reconocimientos de los sitios estudiados se efectuaron mediante inspecciones oculares, que luego dieron paso alos levantamientos topográficos respectivos, en los cuales se empleóuna estación total Topcon modelo GTS-229, con puntos de control registrados en el sistema de coordenadas UTM WGS 84 mediante una unidad GPS Garmin modelo Etrex. Después, considerando el mejor estado de conservación de los edificios prehispánicos en El Obraje 02, se procedió a seleccionar un subconjunto de diez de ellos, tomando en cuenta su cercanía, asociación espacial y estado de conservación. Estas estructuras fueron, entonces, objeto de levantamientos arquitectónicos (planta, cortes y elevaciones) y registros fotográficos en detalle, con el uso de brújulas, winchas, flexómetros y útiles de escritorio varios, además de mangueras de agua para el registro de cotas, y de una cámara Nikon modelo D3100.

El estudio de la tecnología constructiva incluyó una caracterización general de las unidades pétreas empleadas para la edificación de los muros, con la asistencia de especialistas en geología y mineralogía, quienes emplearonreportes y cartas geológicas nacionales $(15,16)$, lentes de aumento y otros implementos. Después se procedió con la recolección de muestras de argamasa arqueológica para los ensayos de laboratorio, utilizandobadilejos, espátulas y brochas, además de bolsas ziplocpara el embalaje de las muestras, fichas de registro de procedencia de las mismas y una balanza electrónica Camry Modelo EK2151 H para el cálculo de la cantidad de material requerido, correspondiente a $30 \mathrm{~g}$.

La identificación y registro de las principales lesiones observadas en las construcciones se efectuó con el apoyo de fichas y de instrumentos varios comogranulómetro, tabla de colores Munsell, lentes de aumento, flexómetros, badilejos, espátulas, brochas, pinceles, escalas gráficas, calibradores, entre otros. Luego, en el gabinete, toda la información obtenida en las fichas se trasladó a tablas en Microsoft Excel, mientras que los levantamientos topográficos y arquitectónicos fueron procesados en AutoCAD. Por su parte, las fotografías fueron editadas en el programa Microsoft Office Picture Manager.

Los ensayos de laboratorio fueron realizados en las instalaciones de la Facultad de Ingeniería Química de la Universidad Nacional del Centro del Perú. Éstos se orientaron a obtener información básica sobre la composición de la argamasa empleada para asentar las piedras que forman parte de los muros de los diez edificios seleccionados en El Obraje 02. Los parámetros analizados fueron en total ocho: porcentaje de humedad, densidad real, $\mathrm{pH}$, presencia de elementos metálicos, porcentaje de carbonatos, porcentaje de materia orgánica, porcentaje de celulosa, y presencia de sulfatos.

\section{RESULTADOS}

\section{Arquitectura y tecnología constructiva}

La arquitectura prehispánica registrada en El Obraje 02 se caracteriza por contar con un sistema espacial conformado por edificios de planta circular, cuyos diámetros internos varían desde los 1,6 m hasta los 4,7m (Tabla $01)$.Estas construcciones carecen de cubiertas, con excepción de dos de las más pequeñas, donde se aprecian indicios de falsas bóvedas de piedra, cuyas alturas son de 1,55 m(edificio 05) y 1,3 m (edificio 07) sobre el suelo actual. En el resto de edificaciones los muros originales tienen alturas variadas y es difícil determinar actualmente el nivel en el que hallaban sus coberturas. 
Tabla 01: Dimensiones y características constructivas de la muestra de los diez edificios estudiados en detalle en el sitio arqueológico El Obraje 02.

\begin{tabular}{|c|c|c|c|c|c|c|c|c|c|}
\hline \multirow{2}{*}{$\begin{array}{l}\text { Nro. } \\
\text { Edificio }\end{array}$} & \multirow{2}{*}{$\begin{array}{c}\text { Diámetro } \\
\text { interno } \\
\text { mínimo } \\
\text { (metros) } \\
\end{array}$} & \multirow{2}{*}{$\begin{array}{l}\text { Diámetro } \\
\text { interno } \\
\text { máximo } \\
\text { (metros) }\end{array}$} & \multirow{2}{*}{$\begin{array}{l}\text { Espesor } \\
\text { promedio } \\
\text { de muros } \\
\text { (metros) }\end{array}$} & \multicolumn{3}{|c|}{ Forma de unidades pétreas } & \multicolumn{3}{|c|}{ Tamaño de unidades pétreas } \\
\hline & & & & Cimiento & Muro & Cubierta & Cimiento & Muro & Cubierto \\
\hline 01 & 2,95 & 3,45 & 0,40 & $\mathrm{Aa}$ & $\mathrm{Aa}$ & n.d. & M & $\mathrm{Pm}$ & n.d. \\
\hline 02 & 3,40 & 3,70 & 0,37 & $\mathrm{Aa}$ & $\mathrm{Aa}$ & n.d. & $\mathrm{Mg}$ & $\mathrm{Pm}$ & n.d. \\
\hline 03 & 2,25 & 2,90 & 0,41 & $\mathrm{Aa}$ & $\mathrm{Aa}$ & n.d. & M & $\mathrm{Pm}$ & n.d. \\
\hline 04 & 1,75 & 2,30 & n.d. & $\mathrm{Aa}$ & n.d. & n.d. & M & n.d. & n.d. \\
\hline 05 & 1,87 & 2,50 & n.d. & n.d. & $\mathrm{Ra}$ & $A$ & n.d. & $\mathrm{Mg}$ & M \\
\hline 06 & 2,95 & 3,40 & 0,48 & $\mathrm{Aa}$ & $\mathrm{Aa}$ & n.d. & $\mathrm{Pm}$ & $\mathrm{Pm}$ & n.d. \\
\hline 07 & 2,20 & 2,30 & n.d. & n.d. & $\mathrm{Ra}$ & $\mathrm{Aa}$ & n.d. & $\mathrm{Pm}$ & $\mathrm{Pm}$ \\
\hline 08 & 4,50 & 4,70 & 0,46 & $\mathrm{Ra}$ & $\mathrm{Ra}$ & n.d. & $\mathrm{Pm}$ & $\mathrm{Pm}$ & n.d. \\
\hline 09 & 3,00 & 3,25 & 0,50 & $\mathrm{Ra}$ & $\mathrm{Ra}$ & n.d. & $\mathrm{Pm}$ & $\mathrm{Pm}$ & n.d. \\
\hline 10 & 1,60 & 2,20 & 0,40 & $\mathrm{Ra}$ & $\mathrm{Ra}$ & n.d. & $\mathrm{Pm}$ & $\mathrm{Pm}$ & n.d. \\
\hline
\end{tabular}

n.d. = Sin datos disponibles a consecuencia de deterioro 0 alternación reciente.

$A=$ Alargada

$\mathrm{Aa}=$ Alargada y angular

$\mathrm{Ra}=$ Redondeada $\mathrm{y}$ angular

$\mathrm{Pm}=$ Pequeño $(10 \mathrm{~cm}-25 \mathrm{~cm}) \mathrm{y}$ mediano $(26 \mathrm{~cm}-40 \mathrm{~cm})$

$M=$ mediano $(26 \mathrm{~cm}-40 \mathrm{~cm})$

$\mathrm{Mg}=$ mediano $(26 \mathrm{~cm}-40 \mathrm{~cm})$ y grande (más de $41 \mathrm{~cm}$ )

El Obraje 02 se encuentra en la ladera de una estribación montañosa de pendiente fuerte, que alcanza los $25^{\circ}$ de inclinación. Por esta razón los edificios se ubican en planos horizontales a diversas alturas, asociados a pequeñas áreas exteriores parecidas a terrazas, con las cuales se conectan mediante vanos de ingreso a modo de puertas, cuyos anchos varían desde los 0,7 m hasta alrededor del metro (Figura 04). Las orientaciones de estas puertas no mantienen un patrón constante, sino que más bien parecen estar en función de la topografía del lugar. Del mismo modo, no hay evidencias de ventanas u otro tipo de aberturas, lo cual crea espacios totalmente cerrados con un solo ingreso. Esto aísla visualmente el interior de los edificiosy restringe el ingreso de luz natural, al mismo tiempo que permite acumulación de calor en el día, con el fin de mitigar el descenso de la temperatura durante la noche.

Por otro lado, los espacios libres están determinados en función de las formas y las orientaciones de los edificios que se

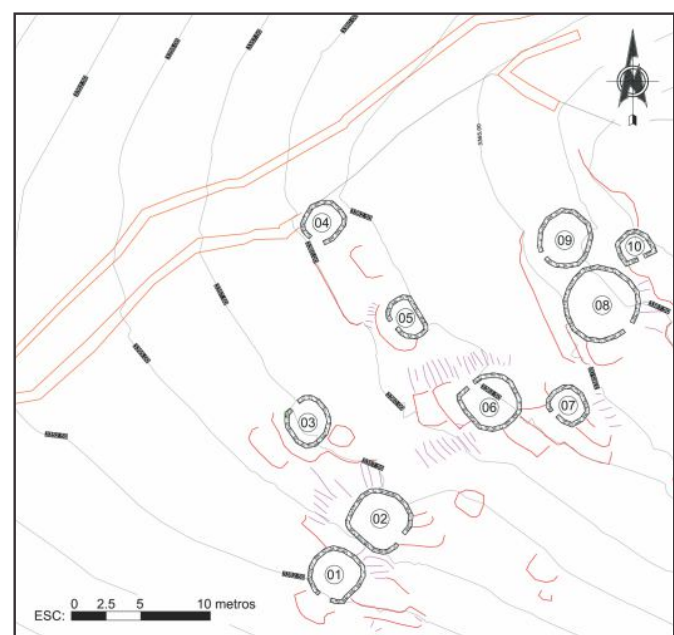

Figura 04: Planta de la muestra de los diez edificios estudiados en detalle en el sitio arqueológico El Obraje 02, mostrando su sistema espacial (Esc. 1:250, intervalo de curvas de nivel: $2,50 \mathrm{~m}$ )

pretende enlazar, lo cual genera una organización espacial agrupada espontánea, basada en la proximidad de las estructuras y en las características del relieve del terreno. Por esta razón no se aprecia una jerarquía espacial clara que origine principios ordenadores precisos y definidos a nivel de todo el conjunto analizado.

Las vías originales de acceso a El Obraje 02 no han logrado ser identificadas sino únicamente uncamino de herradura utilizado hoy por los pobladores del lugar 
para trasladarse hacia las partes altas del territorio del distrito de San Agustín de Cajas. Este sendero recorre el lado noroeste del sitio y genera una aproximación oblicua ascendente con el mismo, además de un contacto visual con los edificios arqueológicos, dando poco a poco una idea de la magnitud del conjunto. Sin embargo, luego de esta aproximación, no encontramos un rasgo definido que separe los espacios hacia el exterior y el interior del sitio, observándose más bien una integración de éste con su entorno.

El sistema de circulación en El Obraje 02 tampoco presenta un patrón definido y más bien es la topografía la que determinalas relaciones entre el desplazamiento y el espacio (Figura 05). De este modo se ha identificado un recorrido curvilíneo que constituye el eje de la circulación del sitio debido a su longitud y posición casi central en relación a los edificios. Este eje recorre el exterior de las estructuras, sin alterar la integridad de cada espacio, y permite la ramificación de recorridos más pequeñoso secundarios, que conducen a otros espacios libres. Cabe agregar, además, que no se han identificado plazoletas o áreas libres, sino que todo el sistema de circulación fluye de manera continua y flexible, incluso hasta integrar la relación espacial interior-exterior como una sola unidad.
El sistema estructural utilizado en la construcción de los edificios es el portante, que emplea como principal material constructivo a la piedra en forma de bloques alargados-angulares y redondeados, de tamaño mediano $(26 \mathrm{~cm}-40 \mathrm{~cm})$ y pequeño $(10 \mathrm{~cm}-25 \mathrm{~cm})$, unidos con argamasa de barro, que constituyen muros cuyo espesor oscila entre los $37 \mathrm{~cm}$ y los $50 \mathrm{~cm}$ en promedio, con alturas que pueden llegar a sobrepasar los dos metros (Figura 06). En su mayoría la piedra empleada es de naturaleza metamórfica y de origen local, destacando las pizarras, esquistos y filitas

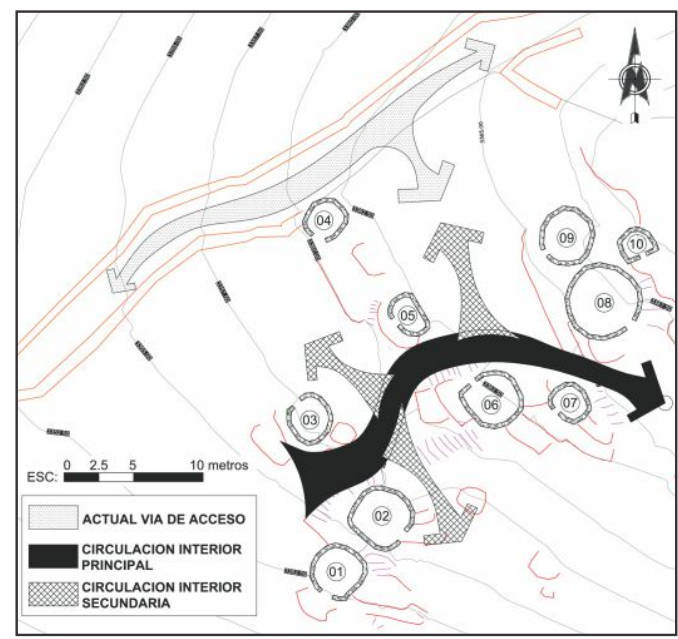

Figura 05: Planta de la muestra de los diez edificios estudiados en detalle en el sitio arqueológico El Obraje 02, mostrando su sistema de circulación (Esc. 1:250, intervalo de curvas de nivel: 2,50 m)

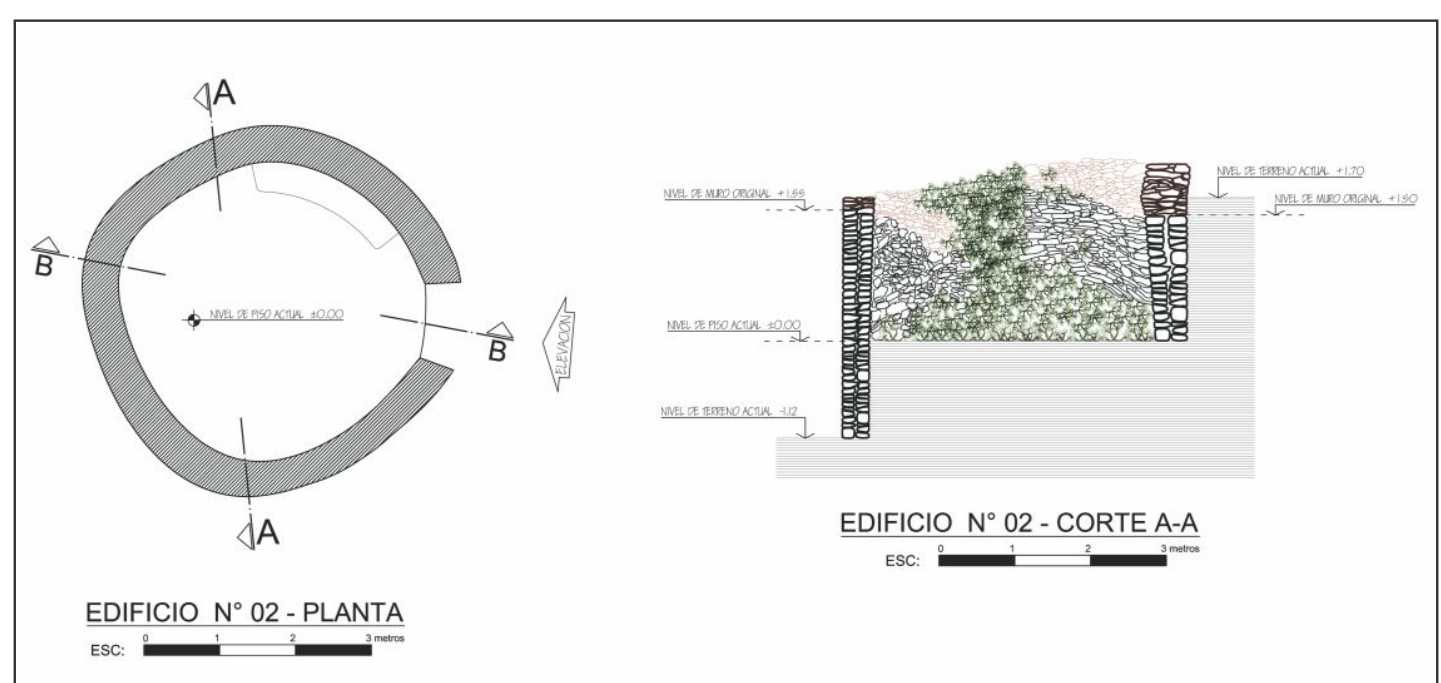

Figura 06: Planta y corte norte-sur (A-A) del edificio 02 en El Obraje 02, mostrando parte de su sistema estructural, los muros originales y los reconstruidos, además de la vegetación existente (Esc. 1:100). 
que datan del Paleozoico y que evidencian presencia de hierro en su composición. Debemos indicar aquí la presencia de un afloramiento rocoso colindante con los edificios 08,09 y 10, que pudo haber sido explotado como cantera.

En el proceso constructivo los bloques o unidades pétreas que acabamos de mencionar fueron desbastados y luego dispuestos en los cimientos, muros y cubiertas en forma encimada, con un ordenamiento rústico. Los muros, que hoy carecen de revestimiento alguno, fueron levantados con una doble hilera de unidades pétreas asentadas con una argamasa de barro de granulometría media ( $1 / 16$ a 2 mm de diámetro) a gruesa (más de $2 \mathrm{~mm}$ de diámetro), cuyas partículas están mal distribuidas en la matriz. Por lo general estas partículas son sub-angulares y presentan una esfericidad media, con una textura arenosa muy gruesa.

La argamasa previamente descrita presenta tonalidades de color que oscila entre el marrón amarillento (10YR 5/4) y el marrón amarillento claro (10YR 6/4) según el sistema Munsell. En cuanto a sus características (Tabla 02), la mezcla presenta poca humedad, entre $4,1 \%$ y $7,5 \%$, con la excepción de dos casos (edificios 04 y 05) donde sí se observó una humedad relativamente alta, entre $15,0 \%$ y $23,5 \%$. En cuanto a su densidad, los análisis arrojaron resultados en general moderados, que oscilan entre valores de 1,69 y 3,01. Del mismo modo, el pH es también moderado, con valores alrededor del nivel neutro.

Desde el punto de vista de su composición, la argamasa contiene elementos como calcio $(\mathrm{Ca})$, sodio $(\mathrm{Na})$, magnesio $(\mathrm{Mg})$, fierro (Fe) y, eventualmente, potasio (K), además de una presencia moderada de $\mathrm{CaCo} 3$ (carbonato de calcio), con porcentajes entre $11,5 \%$ y $17,8 \%$, a excepción de la muestra del edificio 09, que arrojó un valor de 25,5\%. De la misma forma, también se determinó la existencia de materia orgánica no identificada en porcentajes igualmente moderados, que oscilan entre $2,1 \%$ y $4,8 \%$, de manera similar a lo que ocurre con la presencia de celulosa, que muestra valores porcentuales entre $1,4 \%$ y $4,7 \%$. Cabe indicar que los ensayos químicos practicados no lograron identificar las especies vegetales a las que podría corresponder la celulosa registrada. Adicionalmente, se reportó la presencia de iones de sulfato $\left(\mathrm{SO}^{*}\right)$ dentro de la composición de las muestras analizadas, aunque no sus cantidades.

Tabla 02: Resultados de los análisis de laboratorio de las muestras de argamasa arqueológica de los diez edificios estudiados en detalle en el sitio arqueológico El Obraje 02.

\begin{tabular}{ccccccccc}
\hline $\begin{array}{c}\text { Procedencia } \\
\text { de muestra } \\
\text { (N }{ }^{\circ} \text { Edificio) }\end{array}$ & $\begin{array}{c}\text { \% de } \\
\text { humedad }\end{array}$ & $\begin{array}{c}\text { Densidad } \\
(\mathrm{g} / \mathrm{cm})\end{array}$ & $\mathrm{pH}$ & $\begin{array}{c}\text { Elementos } \\
\text { metálicos }\end{array}$ & $\begin{array}{c}\text { Porcentaje } \\
\text { de } \mathrm{CaCO} 3 \begin{array}{c}\text { Porcentaje Porcentaje Presencia } \\
\text { de materia } \\
\text { orgánica }\end{array}\end{array}$ & $\begin{array}{c}\text { de } \\
\text { celulosa }\end{array}$ & de So4 \\
\hline 01 & 7,5 & 2,3 & 7,5 & $\mathrm{Ca}, \mathrm{Na}, \mathrm{Mg}, \mathrm{Fe}$ & 13,4 & 4,8 & 4,1 & Sí \\
02 & 6,5 & 1,95 & 7,1 & $\mathrm{Ca}, \mathrm{Na}, \mathrm{Mg}, \mathrm{Fe}, \mathrm{K}$ & 16,7 & 3,7 & 3,2 & Sí \\
03 & 4,8 & 2,47 & 6,8 & $\mathrm{Ca}, \mathrm{Na}, \mathrm{Mg}, \mathrm{Fe}$ & 16,2 & 3,8 & 2,8 & Sí \\
04 & 23,5 & 1,69 & 6,9 & $\mathrm{Ca}, \mathrm{Na}, \mathrm{Mg}, \mathrm{Fe}, \mathrm{K}$ & 11,5 & 3,7 & 2,9 & Sí \\
05 & 15,0 & 2,5 & 6,9 & $\mathrm{Ca}, \mathrm{Na}, \mathrm{Mg}, \mathrm{Fe}$ & 17,8 & 2,1 & 1,4 & Sí \\
06 & 6,3 & 2,2 & 6,8 & $\mathrm{Ca}, \mathrm{Na}, \mathrm{Mg}, \mathrm{Fe}$ & 13,4 & 4,6 & 4,0 & Sí \\
07 & 6,1 & 2,42 & 7,0 & $\mathrm{Ca}, \mathrm{Na}, \mathrm{Mg}, \mathrm{Fe}$ & 13,7 & 4,5 & 3,6 & Sí \\
08 & 7,2 & 1,95 & 6,8 & $\mathrm{Ca}, \mathrm{Na}, \mathrm{Mg}, \mathrm{Fe}, \mathrm{K}$ & 14,6 & 6,1 & 5,3 & Sí \\
09 & 4,1 & 2,56 & 7,1 & $\mathrm{Ca}, \mathrm{Na}, \mathrm{Mg}, \mathrm{Fe}$ & 25,5 & 5,7 & 4,7 & Sí \\
10 & 6,4 & 3,01 & 7,4 & $\mathrm{Ca}, \mathrm{Na}, \mathrm{Mg}, \mathrm{Fe}, \mathrm{K}$ & 17,6 & 4,3 & 3,5 & Sí \\
\hline
\end{tabular}




\section{Estado de deterioro y tipos de lesiones}

No se identificaron lesiones físicas en los edificios estudiados en El Obraje 02, sino lesiones mecánicas y químicas. En cuanto a las primeras (Tabla 03), se observaron deformaciones en los cimientos de las estructuras, específicamente pandeos y desplomes en grados moderado, alto y muy alto, así como fisuras de naturaleza estructural, desprendimientos y erosión mecánica en grado moderado. grado bajo en pocas estructuras.

En cuanto a las cubiertas, se registraron pocas lesiones debido a que casi en la totalidad de edificios estos componentes de la estructura están ausentes, con excepción de los edificios 05 y 07, como ya se dijo antes. No obstante, en el caso del edificio 07 se identificarondesprendimientos de grado alto, lo cual pone en riesgo la integridad de las partes aún conservadas de dicha estructura.

Tabla 03: Número de edificios afectados por lesiones mecánicas en la muestra estudiada en detalle en el sitio arqueológico El Obraje 02.

\begin{tabular}{lccc}
\hline $\begin{array}{c}\text { Tipo de } \\
\text { lesión } \\
\text { mecánica }\end{array}$ & $\begin{array}{c}\text { Número de } \\
\text { edificios } \\
\text { afectados } \\
\text { en cimientos }\end{array}$ & $\begin{array}{c}\text { Número de } \\
\text { edificios } \\
\text { afectados } \\
\text { en muros }\end{array}$ & $\begin{array}{c}\text { Número de } \\
\text { edificios } \\
\text { afectados } \\
\text { en cubiertas }\end{array}$ \\
\hline Deformaciones & 3 & 6 & 0 \\
Grietas & 0 & 1 & 0 \\
Fisuras & 1 & 3 & 0 \\
Desprendimientos & 1 & 8 & 1 \\
Erosión mecánica & 1 & 6 & 0 \\
\hline
\end{tabular}

En el caso de los muros, hay mayor presencia de lesiones mecánicas, destacando desprendimientos, deformaciones (alabeos, desplomes y pandeos) y erosión mecánica que, en grados diversos (desde bajo hasta muy alto) afectan en su conjunto a buen número de los edificios de nuestra muestra. Cabe indicarse que las fisuras reportadas son de naturaleza estructural y grado moderado, en tanto adicionalmente se reportaron grietas de
Los principales tipos de lesiones químicas corresponden a eflorescencias en unidades pétreas y en argamasa, así como a alteraciones causadas pororganismos vegetales (Tabla 04). En relación a eflorescencias, tanto en unidades pétreas como en argamasa, éstas se hallan presentes en grados bajo y alto en cimientos y muros de muchos de los edificios estudiados. Mientras tanto, las alteraciones a consecuencia de organismos vegetales

Tabla 04: Número de edificios afectados por lesiones químicas en la muestra estudiada en detalle en el sitio arqueológico El Obraje 02.

\begin{tabular}{lccc}
\hline \multicolumn{1}{c}{$\begin{array}{c}\text { Tipo de } \\
\text { lesión } \\
\text { química }\end{array}$} & $\begin{array}{c}\text { Número de } \\
\text { edificios } \\
\text { afectados } \\
\text { en cimientos }\end{array}$ & $\begin{array}{c}\text { Número de } \\
\text { edificios } \\
\text { afectados } \\
\text { en muros }\end{array}$ & $\begin{array}{c}\text { Número de } \\
\text { edificios } \\
\text { afectados } \\
\text { en cubiertas }\end{array}$ \\
\hline $\begin{array}{l}\text { Eflorescencias en unidades } \\
\text { pétreas }\end{array}$ & 7 & 9 & 2 \\
$\begin{array}{l}\text { Eflorescencias en argamasa } \\
\text { Alteración por organismos } \\
\text { vegetales (plantas) }\end{array}$ & 7 & 9 & 2 \\
\hline
\end{tabular}


fueron reportadas en casi todos los edificios en grados diversos, desde bajo hasta muy alto.

Estas lesiones, presentes en cimientos y muros, consisten principalmente en alteraciones en el color, la apariencia y consistencia de la argamasa debido a la proliferación de plantas en ella.

Los dos únicos edificios que aún conservan restos de sus cubiertas (edificios 05 y 07 ) presentan en ellas eflorescencias (en argamasa y en unidades pétreas) y alteraciones ocasionadas por organismos vegetales. En el caso de las eflorescencias en argamasa y en unidades pétreas, estas lesiones son de grado bajo (edificio 05) y alto (edificio 07), mientras que en cuanto a los daños ocasionados por la acción de plantas, los grados son alto (edificio 07) y muy alto (edificio 05). De este modo, podemos decir que en términos generales, el estado de deterioro es moderado en El Obraje 02. En el caso de El Obraje 01, su estado de deterioro puede considerarse malo si tomamos en cuenta que, como ya se dijo, casi la totalidad de sus edificios han sido destruidos.

\section{DISCUSIÓN}

Siguiendo las propuestas de Kostof (17) y otros autores $(18,19)$, consideramos a la arquitectura como un acto social y, por ende, un fenómeno cultural que puede ser abordado desde una perspectiva antropológica. En este sentido, resulta interesante que el sistema espacial registrado en El Obraje 02 no exprese el desarrollo de la noción de retícula, ni la separación clara entre planos horizontales y verticales. Ello nos lleva a plantear que en este lugar el espacio fue concebido de manera unitaria, expresado en el uso de la planta circular, abstraída más fácilmente de un entorno natural carente de referentes directos de ejes independientes y entrecortados mutuamente, como sí se ha reportado, por ejemplo, en el caso del antiguo Egipto (20).

Por su parte, el sistema de circulación identificado en El Obraje 02, continuo, flexible, e influido por la topografía, nos lleva a pensar en el desarrollo de conceptos de recorrido y temporalidad distintos, probablemente asociados a una noción cíclica del tiempo y del cosmos, según han reportado estudios etnohistóricos para el caso andino (21). Esto contrasta bastante con el sentido de unidireccionalidad que caracteriza el desplazamiento al interior de varios edificios levantados por las antiguas civilizaciones occidentales (20, 22), inspiradas en una concepción lineal de la vida y de la dimensión temporal, tal como propone incluso la tradición judeocristiana.

De otro lado, la ausencia de plazoletas o áreas libres, así como la probable integración entre espacios interiores y exteriores en El Obraje 02, podrían ser indicios de una concepción espacial más bien unitaria de lo privado y lo público, salvo por la aislación visual existente en el interior de los edificios debido a la carencia de ventanas y otras aberturas. En este mismo sentido es igualmente importante señalar que estas características denotan una ausencia de jerarquías espaciales y de recorridos, tal como también ha reportado DeMarrais (4) para los asentamientos prehispánicos tardíos locales del valle de Yanamarca.

El sistema estructural portante presente en los edificios de El Obraje 02 destaca por estar conformado por un conjunto de elementos constructivos jerárquicos e interactuantes que reciben, soportan y transmiten cargas hacia el suelo (cimientos, muros y cubiertas) y que actúan de manera conjunta y articulada en cada edificio (23), como una unidad. Esto es precisamente lo que nos lleva a plantear que en la arquitectura estudiada no existen diferencias entre familias constructivas verticales $y$ horizontales, así como tampoco hay ejes y planos verticales y horizontales diferenciados dentro del sistema espacial. Lo que sí se observa es una coherencia racional entre el planteamiento estructural y 
los criterios de estabilidad y solidez. Por esta razón los edificios más pequeños contaron con cubiertas de falsa bóveda y los más grandes recurrieron a soluciones que hicieron uso, probablemente, de materiales como madera y paja. Algo similar se ha encontrado en los sitios estudiados por DeMarrais (4) en el valle de Yanamarca.

En cuanto a otros aspectos de la tecnología constructiva estudiada, debemos indicar la fuerte influencia ejercida por el relieve del terreno, que obligó a los constructores de los edificios de El Obraje 02 a crear primero superficies planas mediante remoción de suelo y movimiento de tierras, para luego comenzar el proceso constructivo con los cimientos y luego con los muros portantes y las cubiertas. Según han indicado los estudios geológicos, las unidades pétreas empleadas proceden del entorno físico inmediato al sitio o de allí mismo, puesto que existe un gran afloramiento rocoso colindante con los edificios 08, 09 y 10 que pudo haber sido explotado como cantera.

La evidencia previamente descrita sugiere entonces que las unidades pétreas empleadas para la construcción de los edificios en El Obraje 02 fueron desbastadas en el mismo lugar. Allí quizás también se haya preparado la argamasa utilizada para asentar las piedras, la cual debió incluir agregados como cal, alguna forma de yeso y otros materiales que compartan las propiedades de la puzolana, según indica la presencia de carbonatos de calcio y de iones de sulfato en las muestras analizadas en laboratorio.

Considerando la poca extensión del asentamiento,la organización del trabajo para la construcción de los edificios pudo haberse efectuado dentro de una estructura social de base familiar. Al respecto, podemos agregar que desde el punto de vista de la función, las construcciones más pequeñas quizás tuvieron una naturaleza funeraria, según indican fuentes históricas (24) e investigaciones arqueológicas (25) para otras partes de los Andes centrales, en tanto que las de mayor tamaño probablemente hayan sido viviendas. Aquí vale la pena indicar que el edificio 08 , el más grande en todo El Obraje 02, podría corresponder a la casa de una de las familias más importantes del lugar, si tomamos en cuenta algunas referencias de tiempos coloniales (26).Sin embargo, la corroboración de esta idea queda sujeta a verificación mediante excavaciones sistemáticas.

Sobre el estado de deterioro de los sitios, El Obraje 01 se encuentra en una situación crítica, puesto que sus evidencias arquitectónicas mejor conservadas están limitadas a unos cuantos edificios dispersos y segmentos de muros de piedra asentada con argamasa de barro, asociados a planos horizontales que conforman terrazas artificiales. En el caso de El Obraje 02, la arquitectura se encuentra en un estado considerablemente mejor, aunque bastante alterada por las intervenciones inconsultas de parte de la población local. Sin embargo, resulta interesante que allí no se hayan apreciado lesiones físicas, probablemente debido a la solidez y coherencia del sistema estructural aplicado y su idónea tecnología constructiva. Por el contrario, las lesiones mecánicas son las más comunes y han sido causadas por los aditamentos hechos en la reconstrucciónno autorizada del año 2008. Es decir, los muros modernos, bastante mal construidos, son los que están provocando el colapso y deterioro de los muros originales.

En el caso de las lesiones químicas, la situación es casi común a todos los edificios analizados debido a los efectos del paso del tiempo y la exposición a los agentes de la intemperie. Los ensayos químicos realizados no han logrado identificar la causa de las eflorescencias, pero en el caso de la menor presencia de organismos vegetales en muros originales, en comparación con los modernos, podríamos sugerir tentativamente una relación con la existencia de iones de sulfato en la argamasa original. Es decir, debido a que la composición de ésta posiblemente incluye materiales como cal y un tipo de yeso o material con propiedades similares a la puzolana, tales elementos podrían haber limitado la proliferación de plantas, a la vez 
que habrían contribuido en la estabilidad de la mezcla, incluso frente a su exposición al agua.

En conclusión, la arquitectura prehispánica de El Obraje 02 - y por sus características similares probablemente también la de $\mathrm{EI}$ Obraje 01 - muestra evidencias de una racionalidad no occidental asociada quizás a una cosmovisión basada en el mito y no en el logos. A partir de esto se desarrollaron reglas y principios ordenadores propios, agrupados en torno a tipos arquitectónicos cuya naturaleza nos resulta difícil de entender por la falta de registros escritos que den cuenta de ellos directamente de boca de sus autores, así como por el prejuicio etnocentrista que aún domina el quehacer arquitectónico en nuestro medio.

Por su parte, la tecnología constructiva revela un conocimiento extraordinario de técnicas y materiales cuya recuperación debe ser pieza clave para el fomento de buenas prácticas de construcción en las zonas rurales en nuestro país, donde el barro, la piedra y la madera son accesibles, y con los cuales la creatividad del hombre del campo puede conducir a experiencias que reivindiquen la valía de la denominada "arquitectura sin arquitectos" (17).

Finalmente, cabe indicarse que los procesos de deterioro observados en El Obraje 01 y El Obraje 02 muestran que en el país las políticas públicas en materia de preservación y conservación del patrimonio arqueológico no han resultado efectivas hasta el momento. Es urgente, por lo tanto, diseñar estrategias adecuadas de diálogo y coordinación con los distintos sectores de la población, a fin de instruirlosen materias de preservación, mantenimiento, reparación y restauración de sus bienes patrimoniales arqueológicos, sin sobredimensionar el papel que éstos juegan en el desarrollo del turismo. En su conjunto, todo esto hará viable la ejecución correcta y responsable de proyectos de conservación y puesta de valor de nuestros sitios arqueológicos, con la finalidad de insertarlos en actividades económicas que permitan el mejoramiento de las condiciones de vida de las personas que viven en sus alrededores.

\section{Agradecimientos}

A los estudiantes de Arquitectura de la Universidad Continental: Rodrigo Rodríguez, Betsabé García, Susan Torres, Ruth Sedano, Rosymary Cusi, Estefany Obregón, Thalía Mendoza, Wendy Cassana, Carmen Hinostroza, Bryan Salinas y Williams Zuasnabar. Asimismo, el reconocimiento geológico fue realizado por los ingenieros Walter Tuppia y Javier Córdova. El levantamiento topográfico fue dirigido por la Arq. Gloria Paytán, y el registro fotográfico a cargo de Martín Arauzo, y los ensayos de laboratorio bajo la dirección del lng. Jaime Vera.

Nuestro agradecimiento a la subvención otorgada por la Universidad Continental a través del Instituto de Investigación para este estudio; asimismo al Lic. Jair Pérez Bráñez, Director Regional de Cultura, y a Oliver Huamán Oros; esta investigación fue autorizada por el Ministerio de Cultura, mediante Resolución Directoral Nro. 4012012-DGPC-VMPCIC/MC.

\section{REFERENCIAS BIBLIOGRÁFICAS}

1. Instituto Nacional de Cultura del Perú. Documentos fundamentales para el Patrimonio Cultural. Lima: Instituto Nacional de Cultura del Perú; 2007.

2. Browman D. Early Peruvian peasants: the culture history of a central highlands valley. [Tesisparaobtener el grado de PhD]. Cambridge: Harvard University, Department of Anthropology; 1970.

3. D'Altroy T. The cultural setting.En: D'Altroy T, Hastorf C, eds. Empire and domestic economy. New York: Kluwer Academic / Plenum Publishers; 2001. Capítulo 2; pp. 27-53.

4. DeMarrais E. The architecture and 
organization of Xauxa settlements. En: D'Altroy T, Hastorf C, eds. Empire and domestic economy. New York: Kluwer Academic / Plenum Publishers; 2001. Capítulo 6; pp. 115-153.

5. Parsons J, Hastings C, Matos R. Prehispanic settlement patterns in the Upper Mantaro and Tarma drainages, Junín, Peru. Volume I: The TaramaChinchaycocha Region. Ann Arbor: University of Michigan, Museum of Anthropology; 2000. (Memoirs of the Museum of Anthropology, University of Michigan Number 34).

6. Bonnier E. Morfología del espacio aldeano y su expresión cultural en los Andes Centrales. En: Bonnier E, Bischof $\mathrm{H}$, eds. Archaeologica Peruana 2. Arquitectura y civilización en los Andes Prehispánicos. Mannheim: Sociedad Arqueológica Peruano-Alemana / ReissMuseum; 1997. Pp. 28-41.

7. Lavallée D, Julien M. El hábitat prehistórico en la zona de San Pedro de Cajas, Junín. Revista del Museo Nacional 1975; 41:81-119.

8. Perales M. Apuntes sobre el Periodo Intermedio Tardío y la presencia inca en la cuenca alta del río Ricrán, sierra central del Perú. Estudios Atacameños 2005; 29: 125-42.

9. Lavallée D, Julien M. Asto: curacazgo prehispánico de los Andes Centrales. Lima: Instituto de Estudios Peruanos; 1983. (Arqueología/7).

10. Moseley M, Mackey C. Peruvian settlement pattern studies and small site methodology. American Antiquity 1972; $37(1): 67-81$.

11. Burga J. Del espacio a la forma. $2^{\mathrm{a}}$.ed. Lima: CONCYTEC; 1989.

12. Ching F. Arquitectura. Forma, espacio y orden. $11^{\mathrm{a}}$.ed. México: Ediciones Gustavo Gili; 1998.

13. García T. Teoría del diseño arquitectónico. 2ª.ed. México: Editorial Trillas; 1990.

14. Broto C. Enciclopedia Broto de patologías de la construcción [Internet]. 2005. [Acceso el 20 de enero de 2012]. Hallado en: http://www.ebook.dow nappz.com/? page $=$ book\&id $=8827$

15. Mégard F. Geología del cuadrángulo de
Huancayo. Lima: Servicio de Geología y Minería del Ministerio de Fomento, Boletín Nro. 18.

16. Paredes J. Geología del cuadrángulo de Jauja. Lima: Instituto Geológico Minero y Metalúrgico del Sector Energía y Minas, Boletín Nro. 48.

17. Kostof S. Historia de la arquitectura. Madrid: Alianza Forma; 2007. Tomo I.

18. Roth L. Entender la arquitectura. Sus elementos, historia y significado. México: Ediciones Gustavo Gili; 2012.

19. De Solá-Morales I. Arquitectura. En: De Solá-Morales I, Llorente M, Montaner J, Ramon A, Oliveras J. Introducción a la arquitectura. Conceptos fundamentales. 2a.ed. Barcelona: Edicions de la UniversitatPolitècnica de Catalunya; 2006. Capítulo 1, pp. 15-26.

20. Alonso J. Introducción a la historia de la arquitectura. 4ㅁ․ed. Barcelona: Editorial Reverté; 2005.

21. Earls J, Silverblatt I. La realidad física y social en la cosmología andina. En: Actes du XLII Congrès International des Américanistes. Paris; 1976. Pp. 299325. Tomo 4.

22. Norberg-Schulz C. Arquitectura occidental. 4a.ed. Barcelona: Editorial Gustavo Gili; 2001.

23. Franco R, Torres L. Estructuras adaptables. Bogotá: Universidad Nacional de Colombia, Facultad de Artes; 2006.

24. Guaman Poma F. Nueva corónica y buen gobierno. Lima: Fondo de Cultura Económica; 2008. Tomol.

25. Isbell W. Mummies and mortuary monuments. A postprocessual prehistory of Central Andean social organization. Austin: University of Texas Press; 1997.

26. Cobo B. Historia del Nuevo Mundo. Madrid: Biblioteca de Autores Españoles $91-92 ; 1964$ [1653].

Correo electrónico:

mperales@continental.edu.pe

hmarroquin@continental.edu.pe 